\title{
DIE VOOLKERRECHTLICHEN VERTRÄGE DES SULTANATS OMAN
}

\author{
Von JoAchim DÜSTER
}

\section{Vorbemerkung}

In der folgenden Darstellung werden in alphabetischer Reihenfolge die Verträge Omans mit 19 Staaten behandelt, wobei auch zwei nicht mehr bestehende Staaten (Nossi Bé, Sohar) aufgeführt sind. Als 20. Gruppe sind dann die beiden multilateralen Verträge behandelt, an welchen Oman beteiligt ist. In der Darstellung wird die Entstehungsgeschichte jedes Vertrages geschildert, sowie die näheren Umstände und das Schicksal des Vertrages. Das Sultanat Oman wird dabei stets unter dieser seit 8. 8. 1970 allein amtlich verwendeten Bezeichnung angeführt. In den Verträgen selbst wird der Staat sowohl als „Oman“, „Muscat“, als auch als „Muscat \& Oman“ bezeichnet. In der anschließenden Dokumentation werden alle völkerrechtlichen Verträge Omans in chronologischer Reihenfolge angegeben, die bisher bekanntgeworden sind. Für eine Reihe von Verträgen konnte die Quelle bisher noch nicht ermittelt werden. Andere einschlägige Texte wie Verträge oder Gesetzesvorschriften dritter Staaten über: Oman sowie Konzessionen und Vorschriften Omans betreffend auswärtiger Beziehungen sind in der Reihe der angegebenen Dokumente nicht aufgeführt, jedoch in der Darstellung erwähnt.

\section{ABU DHABI ${ }^{1}$}

Unabhängiges Scheichtum am Persischen Golf. Seit dem 6. 3. 1892 durch ein „exclusive agreement" mit Großbritannien verbunden und durch dieses Abkommen verpflichtet, „keinesfalls mit irgendeiner Macht außer der britischen Regierung Abkommen zu schließen oder zu verhandeln". Von britischer Seite wird das Abkommen anscheinend so interpretiert, daß Abu Dhabi trotz dieser Bestimmung außenpolitisch handlungsfähig ist und gültige Verträge schließen kann, sofern Großbritannien sein Einverständnis dazu gibt. In einem Brief des Arabian Dept., Foreign \& Commonwealth Office, London, heißt es dazu: "In this connection, I would add that ... the Government of Abu Dhabi is able to deal directly with foreign states in cases where it is considered appropriate for it to do so."

\section{1) 1872}

Abkommen zwischen Oman und Abu Dhabi über die gemeinsame Verwaltung der Oase Buraimi. Das Abkommen war nötig geworden, nachdem die wahabitischen Truppen im Juni 1869 von den Streitkräften Omans und Abu Dhabis aus Buraimi vertrieben worden waren. $\mathrm{Da}$ in Oman schon im Januar 1871 ein Herrschaftswechsel erfolgte und der Herrscher von Abu Dhabi dem neuen omanischen Herrscher nicht freundlich gesinnt war, kam es erst 1872 auf britische Vermittlung hin zu einer Besserung des Verhältnisses und zum Abschluß dieses Abkommens, das vermutlich erst durch den Text von 1896 ersetzt wurde. Der Text des Abkommens ist nicht bekannt.

1 Grenzverträge sind hier nicht aufgeführt, da darüber keine Unterlagen vorhanden sind. 


\section{2) 1896}

Abkommen zwischen Oman und Abu Dhabi über die Verwaltung der Dhahirah, eines Gebietes im Norden von Oman zwischen der Grenzoase Buraimi und der Stadt Ibri. Der bei den Beduinenstämmen sehr beliebte Herrscher von Abu Dhabi verpflichtete sich, in diesem Gebiet des Sultanats für Ordnung zu sorgen, wofür ihm eine jährliche Zahlung von $\$$ M.T. 3000 garantiert wurde. Der Text des Abkommens ist nicht bekannt.

\section{3) 31. 3. 1968}

In einem Kommuniqué niedergelegtes Übereinkommen zwischen Oman und Abu Dhabi, das u. a. Vereinbarungen über eine gemeinsame Währung, direkte Telefonverbindung zwischen beiden Ländern, kulturelle, technische und wirtschaftliche Zusammenarbeit und öftere Meinungsaustausche zwischen den beiden Herrschern enthält. Ferner bestätigt dieses Abkommen frühere Abkommen über den Grenzverlauf zwischen Oman und Abu Dhabi. Das Kommuniqué wurde anläßlich eines Besuchs des Herrschers von Abu Dhabi in Salalah, der Residenz des Sultans, unterzeichnet und ist in arabischer Sprache abgefaßt. Eine englische Übersetzung befindet sich im Arabian Dept. des Foreign Office in London. Weitere Hinweise auf dieses Abkommen siehe UN.DOC. A/7623/Add. 5 (PART II), $\int 8$ und $\int 15$ (in $\ 8$ wird das Abkommen als "Treaty of friendship and cooperation" bezeichnet) sowie ARAB REPORT \& RECORD, ABU DHABI, June/ July 1969 (Sonderheft des ARR).

\section{AUSTRALIEN (mit Queensland)}

Queensland wurde 1859 von Neusüdwales, das bereits 1842 eine eigene Verfassung erhalten hatte, abgetrennt und erhielt ebenfalls volle Selbstverwaltung. Seit dem 1. 1. 1901 ist Queensland ein Staat im Australischen Bund (Commonwealth of Australia). Zwischen Queensland bzw. Australien und Oman unmittelbar wurden keine Verträge abgeschlossen. Queensland trat dem Vertrag Omans mit Großbritannien vom 19.3.1891 mit Wirkung vom 15.12.1892 - gleichzeitig mit Neufundland (s. d.) - bei (siehe HERTSLET'S, Bd. 19, S. 751, Fußnote). Als Queensland am 1. 1. 1901 dem Commonwealth of Australia als Bundesstaat beitrat, übernahm das Commonwealth of Australia das zwischen Queensland und Oman bestehende Vertragsverhältnis. Das Vertragsverhältnis wurde vom Commonwealth of Australia in Úbereinstimmung mit den Bestimmungen des Vertrags von 1891 (s. d.) und der folgenden Verträge mit Wirkung vom 3. 12.1923 gekündigt. In der Verfassung vom 11. 2. 1923, dem letzten Vertrag, dem auch das Commonwealth of Australia indirekt angeschlossen war, heißt es ausdrücklich: "It is also understood that it shall be open to the Dominion of Canada and Commonwealth of Australia to withdraw from the aforesaid treaty at any time on notice being given to that effect by His Majesty's representative at Muscat." Das Commonwealth of Australia beendete das Vertragsverhältnis mit Oman mit Wirkung vom 3. 12. 1923, als der brit. Konsul in Muscat in einem Brief No. 6 auf Verlangen der Regierung das Commonwealth of Australia der omanischen Regierung davon Mitteilung macht. Die Kündigung des Vertragsverhältnisses wird in den State Papers Bd. 117, S. 335, in einer entsprechenden Notiz vermerkt. 
Der "withdrawal“ des Commonwealth of Australia wird in der Vertragsregelung v. 11. 2. 1924 ausdrücklich dokumentiert: „It is also understood that the Commonwealth of Australia have withdrawn from participation in the aforesaid Treaty as per notice given by His Majesty's representative at Muskat in his letter No. 6, dated the 3rd December, 1923.” Insgesamt bestanden also vom 15. 12. 1892 bis zum 3. 12. 1923 vertragliche Beziehungen zwischen Oman auf der einen und Queensland bzw. dem Commonwealth of Australia auf der anderen Seite.

\section{BAHRAIN}

Unabhängiges Inselscheichtum im Persischen Golf. Stand seit 1700 verschiedentlich unter omanischer Herrschaft. Bis 1856 beabsichtigten die omanischen Herrscher immer wieder, die Insel ihrem Herrschaftsbereich einzuverleiben und befanden sich damit in Konkurrenz zu den Wahabiten und den Persern, während Großbritannien mit Erfolg diese Mächte gegeneinander ausspielte und die Unabhängigkeit des Scheichtums sicherte. Seit dem 13. 3. 1892 ist Bahrain durch ein „exclusive agreement" mit Großbritannien verbunden (sieht Abu Dhabi).

\section{1) März 1820}

Vertrag zwischen den Herrschern von Bahrain und Oman, in dem Bahrain die Oberherrschaft Omans über Bahrain anerkennt und sich zu einem jährlichen Tribut von \$M.T. 30000 verpflichtet. Bedingung für diese jährliche Abgabe ist, daß die britische Regierung ein Garantieversprechen für diesen Vertrag abgibt. Als die britische Regierung 1821 noch immer keine Garantieerklärung für die Einhaltung des Vertrags abgegeben hatte, wurde die jährliche Abgabe von den Scheichs von Bahrain mit Duldung Omans auf \$M.T. 18000 herabgesetzt. Der Vertrag ist in arabischer Sprache abgefaßt und wurde im März 1820 von Saiyid Said, dem omanischen Herrscher, und Saiyid Abdul Jalil, dem Bevollmächtigten Bahrains, unterzeichnet. Der Text des Abkommens ist nicht bekannt, er dürfte sich aber im Public Record Office oder im Commonwealth Relations Office in London finden lassen. Die Hauptpunkte des Vertrags sind in einem Brief "Imam of Muscat to General Keir, no date, ca. March 1820" enthalten. Für den Text dieses Briefes siehe BRITISH FOREIGN OFFICE PAPERS 248/40 und/oder FACTORY RECORDS OF THE EAST INDIA COMPANY, Vol. XXXIV, enclosure in a secret letter, April 17, 1820. Weitere Hinweise siehe die entsprechenden Kapitel in Fereydoun Adamiyat, BAHREIN ISLANDS, Praeger, New York 1955.

\section{2) 2.12. 1829}

Friedensvertrag zwischen Bahrain und Oman, in dem Oman die Unabhängigkeit Bahrains anerkennt. Zum Abschluß dieses auch für Oman nicht unvorteilhaften Friedensvertrages war es gekommen, nachdem Bahrain 1828 die Zahlungen an Oman eingestellt hatte, die im Vertrag von 1820 vereinbart worden waren, und Oman im November 1828 vergeblich versucht hatte, Bahrain durch omanisches Militär zu unterwerfen und zu besetzen. Das gespannte Verhältnis zwischen Oman und Bahrain nach dem Krieg vom Nov. 1828, das sich wahrscheinlich nachteilig auf den umfangreichen Handel Omans mit Bahrain auswirkte, wurde durch den Friedensvertrag vom 2. 12. 1829 beseitigt. Der Text des Vertrags ist unbekannt. 


\section{DEUTSCHLAND (BRD)}

1) 19.6. 1967

Schriftwechsel zwischen Deutschland und Oman zur Aufnahme und Regelung konsularischer Beziehungen. Die Einrichtung konsularischer Beziehungen zwischen Deutschland und Oman war nötig geworden, nachdem deutsche Firmen, insbesondere die Wintershall AG, die Gelsenberg AG, die DEA und die Deutsche Schachtbau- und Tiefbohr AG, in Oman tätig geworden waren und dort Kapital investierten. Die Vereinbarung über die Aufnahme konsularischer Beziehungen trat am 19. 6. 1967 in Kraft. Mit der Wahrnehmung der konsularischen Aufgaben wurde auf deutscher Seite am 12. 7. 1967 der damalige deutsche Konsul und spätere Botschafter in Aden beauftragt. Oman läßt seine konsularischen Belange in Deutschland vorerst von der Kgl. Britischen Botschaft in Bonn mit wahrnehmen. Der Text des Briefwechsels wurde nicht veröffentlicht.

\section{2) $27.12 .1968 / 21.5 .1969$}

Schriftwechsel zwischen Deutschland und Oman bezüglich der Regelung der konsularischen Beziehungen, die seit dem Abkommen von 1967 zwischen beiden Staaten bestehen. Darin wird vereinbart, daß die konsularischen Aufgaben im Sultanat Oman zukünftig vom deutschen Konsul in Kuwait wahrgenommen werden sollen. Der Briefwechsel stellt eine Notifikation gemäß Art. 7 des Wiener Übereinkommens über konsularische Beziehungen vom 24. 4. 1963 (BGBl Teil II 1969, S. 1585) dar; er wurde nicht veröffentlicht. Aufgrund dieses Briefwechsels wurde VLR N. Montfort am 2. 2. 1970 durch einen Erlaß des Ausw. Amtes zum deutschen Konsul für Oman mit Sitz in Kuwait ernannt.

\section{FRANKREICH}

\section{1) 1790}

Brief des omanischen Herrschers an den französischen König mit einer Grundsatzerklärung zum omanisch-französischen Verhältnis. Unter anderem heißt es in dieser Erklärung: "Les sentiments qui unissent les Arabes et les Français sont anciens. Ils ne feront que s'accroitre de jour en jour. De tout temps, nous avons distingué la nation française parmi toutes celles de l'Europe. Notre attachement pour elle est si solide que nous disons que nos biens, nos ports et nos vaisseaux sont les siens. Tout est commune entre elle et nous. Il n'éxiste et n'éxistera, jamais pour nous et les français ni distinction de proprietés, ni division d'intérêts... Nous verrons toujours avec un nouveau plaisir les navires et les officiers, de Votre Majesté aborder sur nos Côtes et fréquenter le port de Mascate avec la même confiance que les Côtes et les ports de Votre Empire ... Au surplus, puisse l'amitié, qui regne entre Votre Majesté \& nous, etre éternelle! »

Dieser Brief ist die Antwort auf ein Schreiben des französischen Königs, das dem omanischen Herrscher von Kapitän Macnamara überbracht wurde, der am 10. 3. 1790 auf der "Thétis" nach Muscat kam. Der geschichtliche Hintergrund wird in MILES, S. 277-278, geschildert, wo abschließend bemerkt wird: "Captain Macnamara then negotiated at Muscat, under instructions from the French Foreign Office, a convention with the ruler of Oman, but it was confined to expressions of mutual friendship and goodwill." Die amtliche franz. Übersetzung und der Ori- 
ginaltext des Briefes sind in den Archives Diplomatiques des Ministère des Affaires Etrangères in Paris zu finden und tragen die Stempelnr. 256. Dort befindet sich auch ein Brief des franz. Konsuls in Bagdad vom 28. 5. 1790 (Stempelnummer 70) an den Compte de la Luzerne, in dem die Bedeutung des Dokuments erläutert wird.

\section{2) 16.6 .1807}

Grundlegender Freundschafts- und Friedensvertrag zwischen Oman und Frankreich, bestehend aus einer Präambel und 12 Artikeln. Der Vertrag regelt insbesondere die Handelsbeziehungen und die Konsequenzen, die für den omanischen Handel daraus entstehen, daß zwischen Frankreich und Großbritannien Krieg herrscht. Im Artikel 10 erhält Frankreich das Recht, einen Vertreter in Muscat oder einer anderen Stadt des omanischen Herrschaftsgebietes zu bestellen. Für alle wichtigen Handelsrechte enthält der Vertrag reziproke Meistbegünstigungsklauseln. Die omanische Seite ist jedoch insofern benachteiligt, als der Handel mit blockierten Häfen in Indien und der Handel mit Waffen und Munition in englischen Häfen eingeschränkt wird. Unterzeichnet wurde der Vertrag am 16.6.1807 auf der Insel Mauritius von Decaen, Napoleons Generalbevollmächtigtem für die Gebiete östlich des Kaps der Guten Hoffnung, und von Saiyed Majid bin Khalfan, dem Gesandten des oman. Herrschers, und trat vermutl. sofort in Kraft. Der franz. Text des Vertrags befindet sich in den Archives Diplomatiques du Ministère des Affaires Etrangères in Paris.

\section{3) 17.6 .1808}

Vertrag, bestehend aus Präambel und 5 Artikeln, zur teilweisen Änderung des Vertrages von 1807. Die Änderungen waren von oman. Seite gewünscht worden, da einige Handelsbeschränkungen des Vertrags von 1807 den Handel zw. Oman und Indien schädigten. Frankreich willigte in die Änderung ein, obwohl dadurch Napoleons Blockadebestimmungen für Indien verletzt wurden. Unterzeichnet von Decaen und Abdul Cahir al-Majid auf Mauritius. Text und Fundstellen wie vorige Nummer.

\section{3a) 1. 4. 1822}

Erlaß des Gouverneurs von Bourbon (Réunion), der den Untertanen des omanischen Herrschers Zollfreiheit für wichtige Waren in französischen Häfen gewährt. Um diese Handelsrechte hatte der oman. Herrscher mehrfach nachgesucht. Der amtl. franz. Text der Anordnung findet sich im BULLETIN OFFICIEL DE L'ILE DE BOURBON, 1820-1824, S. 259. Der Erlaß hatte vom 1. 4. 1822 an Gültigkeit. Der Erlaß und der vorausgehende Briefwechsel zwischen Oman und Frankreich führte dazu, daß von manchen Historikern fälschlich die Existenz zweier Handelsverträge vom 30. 3. 1817 und 30. 3. 1822 zwischen Oman und Frankreich angenommen wurde (s. GRAY, S. 180-181, Miles und SAID-RUETE. Dazu und zum Erlaß vom 1. 4. 1822 nahm die Direction des Archives Diplomatiques, Ministère des Affaires Etrangères, Paris, in einer einem Brief v. 11. 12. 1970 angefügten Note wie folgt Stellung: «NOTE sur les traités de 1817/1822 entre la France et le Sultanat de Mascate. - Il n'y aurait pas eu de traité conclu le 30 mars 1817, puisque par une lettre datée d'août 1817 le gouverneur de l'île Bourbon s'excuse auprés de l'Imam de n'avoir pas pu exonérer les marchandises importées par un navire arabe, mais ajoute qu'il envisagerait favorablement la question s'il y avait réciprocité. / L'exemption des droits sur la girofle fut accordée par un 
simple papier de douanes le 30 avril 1818./Le 17 mars 1822 arriva à St.-Denis un navire de Mascate qui demanda à être exonéré. Le 23 mars, le gouverneur rendit compte au Ministre des Affaires Etrangères qu'il avait l'intention d'accorder l'exemption de taxes à l'importation. Cela fut fait par une ordonnance locale du 1er Avril 1822, publié dans le Bulletin officiel de l'Ile Bourbon (1820-1824).»

\section{4) 17. 11.1844}

Grundlegender Freundschafts- und Handelsvertrag zwischen Oman und Frankreich, durch den alle vorhergehenden Abmachungen aufgehoben werden. Der Vertrag gleicht in Inhalt und Form den Verträgen mit den USA v. 1833 und Großbritannien v.1839 und setzt sich aus einer Präambel sowie 19 Artikeln zusammen. Der Vertrag enthält auch Bestimmungen für eine exterritoriale Jurisdiktion des franz. Konsuls. Die Ratifikationen zu diesem Vertrag wurden am 4. 2. 1846 ausgetauscht, und der Vertrag trat damit in Kraft. Der Vertrag wurde in einer franz. und in einer arab. Version unterzeichnet. Hinweise auf den Vertrag sind zahlreich; siehe u. a. MILES; GRAY, S. 184-185; PHILLIPS, S. 105; LANDEN, S. 75; und UN DOC. A/5846, \410

Memorandum unterzeichnet beim Austausch der Ratifikationsurkunden zu diesem am 4. 2. 1846 zwischen M. Desfossés und Saiyid Said bin Sultan, dem omanischen Herrscher. Enthält die Erklärung der franz. Seite, daß sich Art. 17 des Vertrags nur auf den Bereich des Handels erstreckt und die Erklärung der omanischen Seite, daß sie den gesamten Wortlaut des arab. Vertragstextes als verbindlich anerkennt.

Vergl. Gesetz der franz. Republik v. 8. 7. 1852 zur Regelung der exterritorialen Jurisdiktion des franz. Konsuls in Oman, die diesem im Art. 4 und 6, des Vertrages von 1844, zugestanden wurde. Über das Gesetz wurde am 25. 6.1852imCorps Législatif beraten. Es wurde angenommen und an den Senat weitergeleitet, der es am 5. 7. 1852 billigte, so daß es am 8. 7. 1852 vom Präsidenten der Republik unterzeichnet werden konnte. Franz. Text siehe State Papers Bd. 72, S. 243-247.

4a) 10. 3. 1862

Vertrag bzw. gemeinsame Erklärung der Regierungen Großbritanniens und Frankreichs vom 10.3.1862, unterzeichnet in Paris in franz. und englischer Sprache. Großbritannien und Frankreich verpflichten sich, die Unabhängigkeit „of His Highness the Sultan of Muscat and of His Highness the Sultan of Zanzibar" zu respektieren. Dieser Vertrag bedeutet die internationale Anerkennung des sog. Canning Award (vergl. auch LANDEN, S. 201, 209, 222, 274-277, 279-280 und 288; BADGER; WILSON, S. 234; PHILLIPS, .S 135-136), der Omanisch-Ostafrika von Oman trennte und zu einem selbständigen Staat machte. In bezug auf Zanzibar wurde der Vertrag 1890 für nichtig erklärt, um Großbritannien den Abschluß eines Protektoratsvertrages mit Zanzibar zu ermöglichen. In bezug auf Oman ist der Vertrag de jure noch heute gültig, jedoch ohne praktische Bedeutung. Text: State Papers, Bd. 57, S. 785; AITCHISON 1, Bd. 7, S. 105.

\section{4b) 13. 10. 1904}

Abkommen zwischen Großbritannien und Frankreich, in welchem diese Mächte übereinkommen, die strittige Frage der Verleihung der franz. Flagge an omanische Schiffe und die damit eingeschränkten Jurisdiktionsrechte des omanischen Herrschers über diejenigen seiner Untertanen, die damit zu franz. "protégés“ erklärt wurden, vor den Internationalen Gerichtshof in Den Haag zu bringen. 
Das Abkommen nimmt auf den Vertrag von 1862 Bezug, den Großbritannien dahingehend interpretiert, daß er das franz. Vorgehen untersagt. Ferner beziehen sich die vertragschließenden Parteien auf den Art. 1 der „entente cordiale“ vom 14. 10. 1903, der für Konfliktfälle die Lösung durch Verhandlungen bzw. Schiedsgerichte vorsieht. Die Entscheidung des Gerichtshofs von Den Haag soll als endgültig angesehen werden. Der Vertrag besteht aus einer Präambel und 5 Artikeln. Datum des Austausches der Ratifikationsurkunden siehe unter Text des Vertrags siehe State Papers, Bd. 98, S. 46.

4c) 13. 1.1905

Ergänzungsabkommen zum vorigen Abkommen, in dem Großbritannien und Frankreich vereinbaren, die Zeiträume für die Zusammenstellung der dem Internationalen Gerichtshof vorzulegenden Dokumente usw. zu verlängern. Die Ratifikationen zu beiden Verträgen wurden am 18. 1. 1905 in London ausgetauscht. Text in State Papers, Bd. 98, S. 47.

4d) 8. 8. 1905

Schiedsspruch des Internationalen Gerichtshofes von Den Haag über die Frage der Verleihung der franz. Flagge an omanische Schiffe. Der Schiedsspruch wird im wesentlichen den brit. Forderungen gerecht und bestätigt ausdrücklich die Jurisdiktionsrechte des Sultans. Text siehe State Papers, Bd. 98, S. 113-118; Textauszüge siehe WILSON, S. 243, und UN DOC A/5846, \ 420. Hinweise zu den Dokumenten siehe WILSON, S. 240-243; LANDEN, S. 255, 259 und 389.

\section{4e) 4. 2. 1914}

Notenwechsel zwischen Großbritannien und Frankreich über die Beseitigung des illegalen Waffenhandels in Muscat. Frankreich macht darin das Zugeständnis, die durch den Vertrag von 1844 erhaltenen Sonderrechte in Oman nicht in Anspruch zu nehmen, sofern dies die Ausführung der Gesetze und Verträge des Sultanats zur Unterdrückung des illegalen Waffenhandels behindern würde. Frankreich garantiert ferner, daß es sich der Anwendung des Edikts des Sultans vom 4. 6. 1912 (in Kraft getreten am 12. 9. 1912) auf franz. Untertanen nicht mehr widersetzen wird. Das Edikt enthält ebenfalls Bestimmungen über den Waffenhandel. Frankreich erhält dafür die brit. Versicherung, daß Großbritannien seinen Einfluß aufbieten wird, um sicherzustellen, daß franz. Staatsangehörigen im Sultanat die gleiche Behandlung zuteil wird wie brit. Staatsangehörigen. Text: State Papers, Bd. 107, S. $367=$ TS (UK) 1914, Nr. 9.

\section{4f) März 1898}

Konzessionsvertrag, in welchem Frankreich das Recht erhielt, den Hafen von Bandar Jissah südlich von Muscat zu einer Kohlenstation auszubauen, wie das Großbritannien schon 1877 in Muscat getan hatte. Abgeschlossen zwischen Sultan Faisal und dem franz. Vice-Consul M. Ottavi im März 1898. Daraufhin protestierte Großbritannien unter Hinweis auf das Übereinkommen vom 20. 3. 1891 (betr. Nichtabtretung von Gebiet) gegen die Konzession und forderte ihre Annullierung, da befürchtet wurde, die Franzosen würden den dazu gut geeigneten Hafen Bander Jissah zu einer Seefestung ausbauen. Nach militärischem Druck erklärte Sultan Faisal am 16. 2. 1899, daß er die Konzession annulliere. Gleichzeitig forderte er Großbritannien und Frankreich auf, den Konflikt untereinander durch Verhand- 
lungen zu lösen. Der Konflikt wurde endgültig erst 1904 durch die „entente cordiale" beseitigt, nachdem Großbritannien schon im April 1900 mit Frankreich vereinbart hatte, daß letzteres den brit. Kohlenhafen in Muscat benutzen darf. Näheres über die Konzession und den Konflikt siehe LANDEN, S. 248-255; WILSON, S. 239-240; und KUMAR. Text siehe: „Curzon to Hamilton, 25. Febr. 1899, telegram no. 315“, PRECIS ON MUSCAT AFFAIRS, Simla 1906 (Government of India).

\section{GROSSBRITANNIEN}

Die meisten Verträge Omans sind mit Großbritannien geschlossen, das derart enge Beziehungen zu Oman besaß, daß dieses zeitweise als britisches Protektorat bezeichnet worden ist. Diese Vertragsbeziehungen sind daher auch am bekanntesten und in der Literatur am meisten behandelt worden. Aus diesem Grunde und um den Umfang dieser Arbeit nicht allzusehr anschwellen zu lassen, sollen in folgendem die Verträge mit Großbritannien nur kurz vorgestellt werden:

\section{1) Gebietsfragen:}

Durch Vertrag vom 14. 7. 1854 trat Oman die Kuria-Muria-Inseln an Großbritannien $a b$, das dieses durch Vertrag vom 15. 11. 1967 an Oman zurückgab. Durch britischen Schiedsspruch vom 2. 4. 1861 wurde Zanzibar von Oman abgetrennt. Durch Vertrag vom 20. 3. 1891 verpflichtete sich Oman, kein Staatsgebiet abzutreten, welcher Vertrag durch den vom 26. 7. 1958 aufgehoben wurde. Durch Schiedsvertrag Großbritanniens (f. Oman) mit Saudi-Arabien (s. d.) vom 30. 7. 1954 sollte die Frage von Buraimi gelöst werden. Eine einseitige Erklärung SaudiArabiens vom 21. 4. 1866 gegenüber Großbritannien erkannte die Integrität Omans an.

\section{2) Jurisdiktion:}

18. 1.1800 (Installierung eines britischen Agenten), 1873 und 25. 4. 1957 (Jurisdiktion über Inder). Dazu Muscat Orders in Council vom 4. 11. 1867, 3. 2. 1915 6 (m. Änderung vom 1. 4. 1922), 25. 7. 1939, 29. 3. 1949, 28. 10. 1955 (m. Änderung vom 26. 1. 1958, 15. 6. 1959, 25. 1. 1962, aufgehoben durch Order in Council vom 21. 12. 1966).

\section{3) Militärfragen:}

26. 10. 1952 (Waffenstillstand mit Saudi-Arabien, s. d.), 25. 7. 1958 (Stützpunkte usw.).

\section{4) Freundschafts- und Handelsverträge}

12. 10. 1798 (Freundschaft), 31. 5. 1839 (Handel), 19. 3. 1891 (Freundschaft, Handel, Schiffahrt), 5. 2. 1939 (Handel, Schiffahrt), 20. 12. 1951 (Freundschaft, Handel, Schiffahrt). Dazu Omans Konzessionen f. Kohlenstation (1877), Kohlenbergbau (31. 5. 1902), Öl (10.1.1923). Bei dem Vertrag von 1891 handelt es sich um den grundlegenden Vertrag Omans, der Großbritanniens besondere Stellung begründete.

\section{5) Verkehrsfragen}

17. 11. 1864, 19. 1. 1865 betr. Telegrafen, 1934 und 5. 4.1947 betr. Zivilluftfahrt, 25. 7. 1958 Luftfahrt. 


\section{6) Sklaverei-Fragen:}

Verträge vom 4. 9. 1822 (mit Zusätzen vom 31. 5. 1839 und 18. 8. 1845), 2. 10. 1845 , 6. 5. 1850, 14. 4. 1873. Dazu britische Gesetze vom 15. 9. 1848, 5. 8. 1873 (m. Anderung vom 11. 8. 1879) und Order in Council vom 30. 4. 1877.

Die unter Großbritannien aufgeführten Dokumente und Verträge schließen auch die Vertragsverhältnisse Omans zu denjenigen britischen Kolonien und Schutzgebieten usw. ein, die verschiedenen Verträgen zwischen Oman und Großbritannien später durch einen Erlaß der britischen Regierung angeschlossen wurden. Diejenigen britischen Kolonien und Dominions, die aufgrund ihrer weitreichenden Selbstverwaltung Verträgen zwischen Oman und Großbritannien später selbsttätig beigetreten sind bzw. das Vertragsverhältnis später wieder selbsttätig lösten, sind gesondert aufgeführt (s. Irland, Kanada, Australien, Südafrika).

\section{INDIEN}

\section{1) 5. 2.1939}

Beim Abschluß dieses Vertrags war Indien als zu Großbritannien gehöriges Gebiet ausdrücklich als Vertragspartner miteinbezogen. Als Indien unabhängig wurde, übernahm es für kurze Zeit das Vertragsverhältnis. Als Oman den Vertrag fristgerecht auf den 11. 2. 1951 durch eine Mitteilung an die brit. Regierung kündigte, sprach es dabei den Wunsch aus, mit Großbritannien und Indien getrennte Verträge zu schließen. Dies wurde der indischen Regierung im März 1950 von der brit. Regierung mitgeteilt. Am 15. 3. 1953 schloß daraufhin Indien den Vertrag von 1953 mit Oman ab. Dadurch wurde das am 11. 2. 1951 beendete Vertragsverhältnis neu aufgenommen. Vergl. auch UN DOC. A/5846, $₫ 411$ und $₫ 415$.

\section{2) 15. 3.1953}

Grundlegender Freundschafts-, Handels- und Schiffahrtsvertrag zwischen Indien und Oman. Enthält Bestimmungen über Handelsrechte und konsularische Beziehungen. Besteht aus einer Präambel und 11 Artikeln. Der Vertrag wurde am 15. 3. 1953 in drei Versionen (Hindi, Arabisch und Englisch; in Zweifelsfällen ist der engl. Text verbindlich) von Basil Woods Ballard, dem omanischen Außenminister, und Tara Chand, dem außerordentlichen Botschafter Indiens im Iran, unterzeichnet. Der Austausch der Ratifikationsurkunden erfolgte am 14. 2. 1954 in Muscat, worauf der Vertrag laut Art. 10 am 29. 2. 1954 in Kraft trat. Hinweise siehe PHILLIPS, S. 115; und UN DOC. A/5846, \411.

\section{3) 25. 4. 57/10. 3.58}

Durch dieses Abkommen zwischen Oman und Großbritannien, das am 6. 2. 1958 Gültigkeit erklangte und das Abkommen mit Großbritannien vom 20.12.1951 modifizierte, wurde dem Sultan von Oman die Jurisdiktion über Indische Staatsangehörige im Gebiet Omans übertragen. Zuvor hatte der britische Generalkonsul in Muscat bestimmte Jurisdiktionsrechte über Commonwealth-Bürger indischer Nationalițät. Da Indien keine Jurisdiktionsrechte in Oman besitzt, hat der Sultan damit das alleinige Jurisdiktionsrecht über indische Staatsangehörige in seinem Justizbereich. 


\section{IRAN (PERSIEN)}

\section{1) 1798}

Vertrag zwischen Persien und Oman über die Erneuerung des Pachtvertrages über die Hafenstadt Bandar Abbas und das umliegende Gebiet. Die jährliche Pachtsumme, die Oman an Persien zahlen muß, beträgt 6000 Tomans bzw. \$M.T. 15 000. Der Pachtvertrag ist zeitlich unbefristet und gewährt Oman in Bandar Abbas nicht nur alle Zoll- und Handelsrechte, sondern auch andere Hoheitsrechte. Bandar Abbas war von Oman erstmals in neuerer Zeit im Jahr 1780 in Besitz genommen worden (siehe auch VRÜ, 1968, S. 426 oben). Der Vertragstext ist nicht bekannt.

\section{2) April 1817}

Farman des Schahs von Persien, gerichtet an den Herrscher von Oman. Er enthält eine Zusage des Schahs, den Herrscher von Oman bei der Eroberung des Inselscheichtums Bahrain (s. d.) militärisch zu unterstützen. Dieses Übereinkommen hatte der Gesandte Ali bin Abdullah des omanischen Herrschers mit dem Schah in Shiraz ausgehandelt. $\mathrm{Zu}$ der beabsichtigten militärischen Operation kam es jedoch aus verschiedenen Gründen nicht mehr. Der Text findet sich in den BRITISH FOREIGN OFFICE PAPERS, 60/12, als „enclosure in Willock to Earl of Maira, April 6, 1817“. Hinweise auf das Übereinkommen und die vorausgehenden Verhandlungen siehe FREYDOUN ADAMIYAT, BAHREIN ISLANDS, Praeger, New York 1955.

\section{3) 1855}

Order des Schahs von Persien bezüglich der Bedingungen für die erneute Verpachtung von Bandar Abbas an Onan. Sinn und $Z$ weck des Dokumentes sind unklar. Vermutlich handelt es sich um einen Erlaß des Schahs an den Gouverneur von Fars mit Instruktionen für die Verhandlungen mit dem omanischen Herrscher, oder aber um einen neuen Vertragsentwurf, der der omanischen Seite vorgelegt werden sollte. Das Dokument ist ohne Kommentar bei AITCHISON abgedruckt und findet sonst nirgends Erwähnung.

\section{4) April 1856}

Neuer Pachtvertrag zwischen Persien und Oman über Bandar Abbas. Abgeschlossen im April 1856. Dieser neue Vertrag entstand, nachdem Persien entgegen dem Vertrag von 1798 das Gebiet von Bandar Abbas gewaltsam zurückerobert hatte. Der Vertrag ist auf 20 Jahre befristet, die Pachtsumme auf 16000 Tomans heraufgesetzt. Die Hoheitsrechte des omanischen Herrschers werden eingeschränkt; u. a. darf er keine neuen militärischen Stützpunkte in Bandar Abbas errichten und muß dulden, daß in Bandar Abbas die Flagge des Schahs gehißt wird. Der omanische Gouverneur in Bandar Abbas ist ferner zu Hilfeleistungen an die Perser im Kriegsfall verpflichtet.

\section{4a) 17.11. 1856}

Angeblicher Vertrag über die Pacht von Bandar Abbas zwischen Oman und Persien. Enthält etwa die gleichen Bestimmungen wie der Vertrag vom April 1856 und setzt sich aus 10 Artikeln zusammen. Die Pachtsumme wird in Art. 8 jedoch auf nur 14000 Tomans festgesetzt. Dieser Vertrag wird allgemein als eigentlicher 
Pachtvertrag von 1856 angesehen, während der Vertrag vom April 1856 nur wenigen Historikern bekannt ist. Für die Lösung des Problems gilt die Möglichkeit, daß 1. der Vertrag vom April 1856 der einzig authentische und gültige ist, 2. der allgemein bekanntere Vertrag vom 17. 11. 1856 der einzig richtige ist, oder 3. der im Jahre 1856 zuerst der Vertrag vom April abgeschlossen und am 17. 11. 1856 durch diesen Vertrag ersetzt wurde. Die 2. Möglichkeit wird dadurch ausgeschlossen, daß er im Text dieses Dokuments erwähnte omanische Herrscher und Vertragspartner Saiyid Said bereits am 19. 10. 1856 gestorben war, während der Vertrag angeblich vom 17.11. 1856 datiert. In VRÜ 1968, 4. Heft, S. 431, wird behauptet, Oman habe den Pachtvertrag durch Druck auf das schwache Persien erwirkt, was angesichts der schwachen Position Omans in den üblichen Krisen nach dem Tode eines Herrschers nicht denkbar ist.

Die 3. Möglichkeit scheidet aus, da es für sie keinerlei historische Anhaltspunkte gibt und der Text des Dokuments vom 17. 11. 1856 nicht darauf hinweist, daß es zur Erneuerung eines erst kürzlich geschlossenen früheren Abkommens dienen sollte.

Es ist also anzunehmen, daß der ursprüngliche Vertrag der im April 1856 abgeschlossene ist und daß im November 1856 kein neuer Vertrag abgeschlossen wurde.

\section{5) 4. 8.1868}

Vertrag zwischen Oman und Persien über die erneute Verpachtung von Bandar Abbas an Oman. Im März 1866 hatten die Perser Bandar Abbas zurückerobert, nachdem im Februar 1866 in Oman ein Herrscherwechsel erfolgt war. Wegen interner Schwierigkeiten konnte der neue omanische Herrscher Saiyid Salim vorerst nichts gegen Persien unternehmen, bis er im April 1868 Bandar Abbas durch seine Flotte vollständig blockieren ließ und den gesamten Handel lahmlegte, Daraufhin wandten sich die Perser an Großbritannien um Vermittlung. Der britische Political Resident im Persischen Golf erreichte den Abzug der Blockadeflotte und garantierte Oman einen neuen Pachtvertrag, der am 4. 8. 1868 in Shiraz abgeschlossen wurde. Er umfaßt 15 Artikel, ist auf 8 Jahre befristet und setzt die jährliche Pacht auf 30000 Tomans herauf. Art. 12 enthält die später verhängnisvolle Klausel, daß der Vertrag ungültig wird, sobald in Oman ein gewaltsamer Umsturz stattfindet. Als am 1. 10. 1868 aufständische Truppen der konservativen Koalition Muscat besetzten und Saiyid Salim absetzten, kann Persien den Vertrag annullieren. Hinweise siehe Badger, PHILLIPS, S. 140, und MILES.

\section{5a) 13. 1.1898}

Erlaß des Sultans vom 13. 1. 1898 mit sofortiger Wirkung, zum Verbot des Waffenhandels und der Einfuhr von Waffen nach Persien und Indien. Der omanische Herrscher erließ dieses Gesetz auf Wunsch der persischen und britischen Regierungen und im Übereinkommen mit diesen Staaten. Muscat war um die Jahrhundertwende (1900) der Mittelpunkt des Waffenhandels im Gebiet des Indischen Ozeans und Persischen Golfes. Die Proklamation enthält ferner das Zugeständnis an Persien und Großbritannien, omanische Schiffe mit Waffen an Bord zu konfiszieren. 


\section{IRLAND (EIRE)}

Irland hat keine eigenen Verträge mit Oman abgeschlossen. Als Irland nach dem Inkrafttreten des Vertrages vom 6. 12. 1921 mit Großbritannien als Freistaat Irland den Status eines selbständigen Dominions erhielt, übernahm es das durch den Vertrag vom 19. 3. 1891 begründete Vertragsverhältnis. Vermutlich übernahm später der durch die irische Verfassung vom 29. 12. 1937 entstandene unabhängige Staat Eire dieses Vertragsverhältnis, das am 11. 2. 1939 auslief und in bezug auf den Staat Eire nicht erneuert wurde.

In den Dokumenten von 1925 und 1926 zur Verlängerung des Vertrages von 1891 wird ausdrücklich festgestellt: "It is also understood that it shall be open to the Dominion of Canada and the Irish Free State to withdraw from the aforesaid treaty at any time on notice being given to that effect by His Majesty's representative at Muscat." Diese Formel wird in den späteren Verlängerungen stets wiederholt, auch in der letzten vom 27.11.1937, so daß Irland also bis zuletzt Vertragspartner war.

\section{ITALIEN}

Italien hat mit Oman keine direkten Verträge abgeschlossen. Aufgrund eines Abkommens zwischen Großbritannien und Italien (vergl. State Papers, Bd. 96, S. 1131) kam es schließlich dazu, daß Oman mit Großbritannien und Italien übereinkam, letzteren Mächten das „right of search of Muscat vessels“ zu gewähren. Um 1910 war ein Vertreter Italiens mit dem Titel eines "Special Agent“ in Muscat akkreditiert.

17. 10. 1903

Bekanntmachung eines Erlasses bzw. einer Erklärung des Sultans mit sofortiger Gültigkeit. Darin wird die Einfuhr von Waffen und Munition nach den italienischen und brit. Gebieten in Ostafrika untersagt und gleichzeitig britischen und italienischen Schiffen das Recht verliehen, verdächtige Schiffe zu durchsuchen und zu konfiszieren, falls sie gegen den Erlaß verstoßen. Die entsprechende Italien betreffende Klausel der Erklärung lautet: „Furthermore, I hereby grant authority to Italian ships of war also to search suspected Muscat vessels either in territorial waters or on the high seas." Text siehe State Papers, Bd. 96, S. 666-665. Hinweise siehe UN DOC A/5846, Annex VII, $\$ 9$.

\section{KANADA (MIT NEUFUNDLAND)}

Kanada hat keine eigenen Verträge mit Oman abgeschlossen, trat aber dem Vertrag Omans mit Großbritannien vom 19. 3. 1891 bei. Der Beitritt des „Dominion of Canada" erfolgte am 11. 4. 1893 (siehe HERTSLET'S, Bd. 19, S. 751). Das Vertragsverhältnis lief am 11. 2. 1939 aus und wurde in bezug auf Kanada nicht erneuert. Es gilt das gleiche wie oben bei Irland. Es wurde keine Notiz über einen "withdrawal“ Kanadas gefunden. Insgesamt bestanden also vom 11. 4. 1893 bis zum 11. 2. 1939 vertragliche Beziehungen zwischen Kanada und Oman. 


\section{NEUFUNDLAND}

Neufundland erhielt 1855 Selbstverwaltung und bekam im folgenden Zeitabschnitt weitere Rechte bezüglich seiner staatlichen Eigenständigkeit, bis es sich 1948 dem kanadischen Bundesstaat anschloß. Neufundland hat keine eigenen und direkten Verträge mit Oman abgeschlossen. Es trat dem Vertrag von 1891 mit Wirkung vom 15. 12. 1892 bei (siehe HERTSLET'S, Bd. 19, S. 751, Fußnote). Da ein „withdrawal“ Neufundlands von diesem Vertrag und den folgenden Verträgen über seine Verlängerung nirgends verzeichnet ist, darf angenommen werden, daß das Vertragsverhältnis mit Oman erst mit dem Auslaufen des Vertrages am 11. 2. 1939 beendet wurde und daß somit vom 15. 12. 1892 bis zum 11. 2. 1939 vertragliche Beziehungen zwischen Oman und Neufundland bestanden.

\section{NIEDERLANDE}

\section{1) 7. 4.1877}

Handelsvertrag zwischen den Niederlanden und Oman, bestehend aus 3 Artikeln, die reziproke Meistbegünstigungsklauseln im Bereich des Handels und Zolls zum Inhalt haben. Der französische Originaltext wurde am 7. 4. 1877 in Den Haag vom niederländischen Außenminister Van Der Does de Willebois und am 27. 8. 1877 in Muscat von Sultan Turki unterzeichnet und trat am 27. 8. 1877 in Kraft. Er ist bis heute (April 1971) in Kraft. Der Handelsvertrag war von Großbritannien vermittelt worden.

In einem niederländischen Vertragswerk findet sich zu diesem Vertrag noch folgende ergänzende Erläuterung: „Van kracht was op 1 Januari 1911 de Verklaring van 7 April/27 Augustus 1877. Daarbij is bepaald dat Nederlandsche onderdanen, alsook de Nederlandsche scheepvart en de goederen, komende von of voortgebracht in Nederland of zinje kolonien, in Mascate meestbegunstiging zullen genieten, op grond, dat Mascate van Nederlandsche zijde dezelfde behandeling ondervindt. In de Verklaring van 1877 is geen opzeggingstermijn aangegeven." $\mathrm{Zu}$ beachten ist, daß die niederländischen Übersetzungen des franz. Originals oft im Wortlaut voneinander abweichen und mitunter die Präambel vermissen lassen. Es ist daher zweckmäßig, sich stets an den franz. Originaltext zu halten. Hinweise auf den Vertrag siehe UN DOC A/5846, \410.

\section{2) 9. 4.1968}

Briefwechsel zwischen den Niederlanden und Oman, bestehend aus einem Brief des niederländischen Außenministers Luns vom 9. 4. 1968 und einem Brief des Sultans Said bin Taimur vom 25. 8. 1968, über die Aufnahme und Regelung konsularischer Beziehungen zwischen den beiden Staaten. Das Recht zur Ernennung eines Konsuls ist reziprok. Für die niederländische Seite wird festgelegt, daß ein Mitglied der niederländischen Botschaft in Bagdad zum Konsul für Oman ernannt werden soll. Den Konsuln wird in den Gebieten der Vertragspartner volle Bewegungsfreiheit zur Ausübung ihrer dienstlichen Pflichten garantiert. Der Briefwechsel nimmt Bezug auf eine Unterredung des niederländischen Botschafters in Bagdad mit Sultan Said vom Januar 1968 und ist in englischer Sprache gehalten. Das Abkommen trat am 25. 8. 1968 in Kraft und wurde vom niederländischen Außenminister am 22. 5. 1969 zur Veröffentlichung freigegeben. 
In einem Brief des niederländischen Außenministers an den Sultan vom 30.12. 1968 wurde Herr A. H. Croin, Leiter der konsularischen Abteilung der niederländischen Botschaft in Bagdad, zum Konsul für Oman ernannt. Er wurde inzwischen durch W. Hellema, den Geschäftsträger der Botschaft in Bagdad, ersetzt. Oman hat bisher keinen eigenen Vertreter für die Niederlande ernannt.

Mittels eines Telegramms vom 2. 8. 1970 teilte Sultan Qabus, der neue omanische Herrscher seit dem 23. 7. 1970, der niederländischen Regierung mit, daß er die Nachfolge seines Vaters, Sultan Saids, angetreten habe und daß er die zwischen seinem Sultanat und den Niederlanden bestehenden Vertragsverhältnisse respektieren werde. In einem Telegramm vom 11. 8. 1970 dankte die niederländische Regierung Sultan Qabus für dessen Mitteilung und sprach dabei die Hoffnung aus, daß die guten Verhältnisse fortgesetzt würden.

Inzwischen wird die Frage der niederländischen konsularischen Vertretungen aufs neue untersucht.

\section{NOSSI BE}

Nossi Bé war ehemals ein unabhängiges „Königreich“ westlich der Nordspitze Madagascars. Es umfaßt die Insel Nossi Bé und einige kleine Dependenzien. Regiert wurde Nossi Bé von einer Königin, die unter dem starken Einfluß ihrer sogenannten „Minister“ stand. Das „Königreich“ Nossi Bé kam um 1839 unter omanische Schutzherrschaft. Am 5. 3. 1841 (nach anderen Angaben am 14. 7. 1840) schloß dieses omanische Protektorat widerrechtlich einen Vertrag mit Frankreich, in dem das gesamte „Königreich“ Nossi Bé an Frankreich abgetreten wurde. Diplomatische Proteste Omans blieben erfolglos.

\section{1) um 1839}

Protektoratsvertrag zwischen Oman und der Königin von Nossi Bé, Seniku (auch Seneekoo) Tsihometa. Der Vertrag wurde von den Ministern der Königin deshalb abgeschlossen, weil Nossi Bé von Angriffen der Stämme auf der Insel Madagaskar gefährdet war und Hilfe benötigte. Die Königin verpflichtete sich „to surrender all her dominions on the island of Nossi Bé to Said and further agreed to pay him thirty thousand dollars and a duty of five per cent. on all imports from the island". Saiyid Said, der omanische Herrscher, verpflichtete sich dafür "to take charge of the fort and to protect us on the sea side and generally protect us as he does his other subjects". Der omanische Herrscher sandte nach dem Abschluß des Vertrags einige Beamte und eine rote Flagge (die damalige Nationalflagge Omans) nach Nossi Bé und siedelte dort einige Omanis an. Er entsandte jedoch keine Schutztruppe auf die Insel.

Im Sommer 1840 besuchte Kapitän Guillan auf der französischen Korvette "Dordogne" Nossi Bé und bot der Königin einen Protektoratsvertrag an, was diese im Hinblick auf den mit Oman bestehenden Vertrag ablehnte.

Am 11. 11. 1840 erfuhr die omanische Regierung, daß weitere französische Schiffe Nossi Bé besucht hatten und daß französische Soldaten dort eine Festung bauten. Am 5. 3. 1841 unterzeichnete die Königin von Nossi Bé formell einen Vertrag mit Frankreich, in dem sie ihre Gebiete für eine jährliche Rente von 1200 Francs an Frankreich abtrat.

Saiyid Said, der omanische Herrscher, forderte daraufhin Großbritannien auf, in dieser Angelegenheit zu intervenieren. Großbritannien befürchtete aber eine Ver- 
schlechterung des Verhältnisses zu Frankreich und begründete seine Ablehnung damit, daß „there is a material difference between territories which have for a length of time belonged to a Sovereign and districts which have only recently tendered their submission to such sovereign, and over which he has exercised no practical authority". (British Foreign Secretary to Saiyid Said).

Der Text des Vertrags konnte vorerst nicht aufgefunden werden.

\section{PAKISTAN}

1) 8. 4.1958

Abkommen zwischen Oman und Pakistan über die Abtretung der Enklave Gwadar an Pakistan. Das nicht veröffentlichte Abkommen bestimmte, daß die Hafenstadt Gwadar und das umliegende Gebiet vom 8. 9. 1958 an zu Pakistan gehören sollte. Gwadar war 1794 von Oman in Besitz genommen worden und bildete seither einen Teil des Sultanats (vergl. auch VRU 1968, S. 426 oben, wo fälschlich gesagt wird, Gwadar habe nur von 1837 bis 1958 zu Oman gehört). Sultan Said bin Taimur erhielt als Entschädigung eine Zahlung von $\$ 3000000$ und eine Gewinnbeteiligung an eventuellen zukünftigen Erdölfunden im Gebiet von Gwadar.

Das Abkommen war von Pakistan seit 1947 gewünscht worden, wurde aber erst 1958 erzielt. Bei den Verhandlungen zu diesem Abkommen wurde Pakistan von Großbritannien vertreten.

\section{PORTUGAL}

\section{1) Februar 1826}

Grundlegender Handels-, Schiffahrts- und Freundschaftsvertrag zwischen Portugal und Oman, bestehend aus einer Präambel und 9 Artikeln. Alle wichtigen Handelsrechte sind bis auf Art. 3 (Portugiesischer Einfuhrzoll wird auf 10 Prozent ad valorem festgesetzt; in omanischen Häfen beträgt er nur 5 Prozent) reziprok. Der Vertrag ähnelt in Inhalt und Form dem Vertrag Omans mit den USA. Er wurde in portugiesischer und arabischer Version im Februar 1826 in Mozambique vom General-Gouverneur der Kolonie Mozambique, Sebastiao Xavier Bothelho, und dem omanischen Gesandten Saif bin Ahmed bin Said unterzeichnet. Er wurde niemals formell beendet und ist auch zeitlich nicht begrenzt; doch darf man annehmen, $\mathrm{da} ß$ er stillschweigend als bedeutungslos und unanwendbar betrachtet wird.

\section{2) 28. 3.1828}

Grundlegender Handels-, Schiffahrts- und Freundschaftsvertrag zwischen Portugal und Oman. Enthält im wesentlichen die Bestimmungen des Vertrags von 1826 in detaillierter Form, sowie einen Artikel über die Festlegung der Grenze zwischen der Kolonie Mozambique und Omanisch-Ostafrika. Wegen dieser Klausel, deren Inhalt nicht mit den Ansprüchen Omans übereinstimmte, wurde der Vertrag von Oman nicht ratifiziert und erlangte keine Gültigkeit. Text in Mocambique Pasta 4, 1840.

3) 27.5. 1892

Grundlegender Vertrag zwischen Oman und Portugal. Enthält wie der Vertrag von 1828 einen Artikel über die Festlegung der Grenze zwischen der Kolonie 
Mozambique und Omanisch-Ostafrika, diesmal gemäß den omanischen Ansprüchen. Der Vertrag wurde von Portugal wegen dieses Artikels und von Oman wegen des zu eigenmächtigen Handelns des Gesandten, der den Vertrag abschloß, nicht ratifiziert und blieb ungültig. Text in Zanzibar Archives.

4) 20.11. 1843

Kleineres Abkommen zwischen Portugal und Oman bezüglich der Papiere der omanischen Schiffe, die portugiesische Häfen anlaufen. Ergänzt damit den Vertrag von 1826 und wurde anläßlich des Besuchs des portugiesischen Generalgouverneurs von Mozambique in Zanzibar, der zeitweiligen Residenz des omanischen Herrschers, im September/Oktober 1843 ausgehandelt. Unterzeichnet wurde das Abkommen in Zanzibar in einer arabischen Fassung von Saiyid Said, dem omanischen Herrscher, am 29. 9. 1843 und in Mozambique am 20. 11. 1843 vom Sekretär des Generalgouverneurs von Mozambique, Francisco de Moraes, Corrêa de Castro.

\section{SAUDI-ARABIEN (Wahabiten-Reich)}

1) 1803

Auf drei Jahre befristeter Waffenstillstandsvertrag zwischen Oman und dem Herrscher der Wahabiten. Der Amir der Wahabiten verpflichtet sich, drei Jahre lang nichts gegen Oman zu unternehmen und auch keine anderen Stämme gegen den omanischen Herrscher aufzuwiegeln. Ferner erkennt er die Herrschaft von Saiyid Sultan in Oman an. Saiyid Sultan verpflichtet sich dafür zu einer jährlichen Zahlung von \$M.T. 12000 und gestattet dem Amir der Wahabiten, eine ständige wahabitische Gesandtschaft in Muscat einzurichten. Der Vertrag wird nach wenigen Monaten von den Wahabiten gebrochen, deren Truppen über Buraimi zur BatinahKüste nach Oman einmarschieren. Im Oktober 1803 kommt es erneut zu Waffenstillstandsverhandlungen zwischen Oman und den Wahabiten. Während der Verhandlungen stirbt in der Hauptstadt der Wahabiten deren Amir, und der wahabitische Unterhändler muß wegen interner Unruhen im Wahabitenstaat die Verhandlungen abbrechen und sich mit seinen Truppen zurückziehen.

Der Vertrag muß im Frühjahr 1803 abgeschlossen worden sein; der Text liegt nicht vor.

\section{2) Mai 1833}

Vertrag zwischen Oman und den Wahabiten, in dem sich der Amir der Wahabiten verpflichtet, die territoriale Integrität Omans zu respektieren und sich aus Buraimi zurückzuziehen. Dafür verpflichtet sich der omanische Herrscher zu einer jährlichen Zahlung von \$M.T. 5000. Der Vertrag wurde im Mai 1833 abgeschlossen; der Text liegt nicht vor. Um 1837/39 stellte Oman die jährlichen Zahlungen an das Wahabitenreich ein.

\section{3) 1836}

Bündnisvertrag zwischen Oman und dem Kommandanten des zeitweilig in Buraimi stationierten Wahabiten-Heeres, gerichtet gegen das Fürstentum Sohar (siehe dort). Die beiden Parteien vereinbaren, das Fürstentum Sohar zurückzuerobern und die Beute zu teilen, während das Fürstentum Sohar wieder in den Herrschaftsbereich des Herrschers von Oman eingegliedert werden soll. Der Angriff auf Sohar wird zwar durchgeführt, doch läßt der Herrscher von Sohar das Gerücht verbreiten, 
die Wahabiten wollten Sohar für sich erobern und es als Stützpunkt für militärische Operationen gegen den Herrscher von Oman benützen. Daraufhin bricht der omanische Herrscher den Angriff ab, und die wahabitischen Truppen ziehen sich aus Oman zurück.

\section{4) Februar 1845}

Waffenstillstandsvertrag zwischen Oman und dem Wahabitenreich. Er wurde vereinbart, nachdem wahabitische Truppen im Februar 1845 die Oase Buraimi besetzten und von Oman einen jährlichen Tribut von $\$$ M.T. 20000 gefordert wurde. Saiyid Thuwaini, der seinen Vater, den omanischen Herrscher, für die Zeit dessen Abwesenheit vertrat, schloß den Waffenstillstand zur Fixierung des status quo ab, bis er von seinem Vater aus Omanisch-Ostafrika die nötigen Instruktionen erhalten würde, wie der Forderung zu begegnen sei. Der Kommandant der wahabitischen Truppen bricht den Waffenstillstand wenige Wochen später, indem er in die Batinah einfällt und einen Plünderzug unternimmt.

\section{5) 1845}

Vertrag zwischen Oman und dem Wahabitenreich. Auf britischen Druck hin hatte der Wahabitenherrscher seine Forderungen gemäßigt, und es wurde eine jährliche Zahlung Omans an den Amir der Wahabiten von \$M.T. 5000 vereinbart. Der Text des Vertrages liegt nicht vor. Der jährliche Tribut wurde nur für 3-4 Jahre gezahlt.

\section{6) April 1853}

Nach einem weiteren Angriff wahabitischer Truppen gegen Oman wird ein neuer Vertrag z.wischen Oman und dem Amir der Wahabiten von den Briten vermittelt. Der omanische Herrscher verpflichtet sich zu einer jährlichen Zahlung von \$M.T. 12000. Dafür garantiert der Amir der Wahabiten ausdrücklich die territoriale Integrität Omans. Der Text des im April 1853 abgeschlossenen Vertrages ist nicht bekannt.

\section{7) 26. 10. 1952}

Waffenstillstandsvertrag zwischen Saudi-Arabien und Großbritannien, wobei letzteres im Auftrag des Sultans von Oman sowie stellvertretend für Abu Dhabi handelte. Er wurde abgeschlossen, nachdem im August 1952 saudi-arabische Soldaten in Buraimi einmarschiert waren. Daraufhin ließ der Sultan von Oman, in Zusammenarbeit mit dem Imam, der einen "Jihad“ (heiliger Krieg) gegen Saudi-Arabien erklärte, seine Truppen und Stammesstreitkräfte in Stärke von ca. 8000 Mann mobilisieren und auf Buraimi marschieren. Um einen Krieg zu vermeiden, überredete Großbritannien den Sultan zu diesem Waffenstillstandsabkommen, das der Sultan am 10. 10. 1952 „im Prinzip“ akzeptierte und das am 26. 10. 1952 in Jedda unterzeichnet wurde. Als Vermittler hatte der Botschafter der USA in Jedda gedient. Der Waffenstillstand erwies sich später als eine der größten Fehlentscheidungen in der omanischen Geschichte. Am 2. 4. 1953 überbrachte der britische Botschafter in Jedda der saudi-arabischen Regierung eine Note, in der Großbritannien erklärte, es sehe sich gezwungen, den Waffenstillstandsvertrag fortan als ungültig zu betrachten und sich im Namen des Herrschers von Abu Dhabi volle Handlungsfreiheit vorzubehalten. Der Sultan behält sich ebenfalls volle Handlungsfreiheit vor. 
Vertrag zwischen Saudi-Arabien und Großbritannien, letzteres im Auftrag des Sultans von Oman sowie stellvertretend für Abu Dhabi, in welchem beschlossen wird, den Buraimi-Konflikt vor ein unabhängiges Schiedsgericht zu bringen. Der Vertrag, bestehend aus einer Präambel und 20 Artikeln, regelt die Zusammensetzung und Arbeitsweise des Schiedsgerichts und die Interimsverwaltung des strittigen Gebiets. Der Vertrag trat mit dem Datum der Unterzeichnung am 30. 7. 1954 in Kraft. Das daraufhin gebildete Schiedsgericht stellte seine Arbeit am 16. 9. 1955 ein, nachdem das Verhalten Saudi-Arabiens ein ordnungsgemäßes Vorgehen des Tribunals unmöglich gemacht hatte (u. a. durch Bestechungen). Am 26. 10. 1955 wurde daraufhin die Oase Buraimi wieder von Truppen aus Oman und Abu Dhabi in Besitz genommen.

Notenwechsel vom gleichen Tage zwischen dem britischen Botschafter in Jedda und dem saudi-arabischen Außenminister. Der Austausch der Noten, in denen die Bedingungen für die Aufnahme der Schiedsgerichtsverhandlungen niedergelegt und die Grenzen des umstrittenen Gebiets definiert werden, erfolgte im Anschluß an die Unterzeichnung des Vertrags.

\section{SOHAR}

Von 1829 bis 1852 ein autonomes Fürstentum mit eigener Außenpolitik auf omanischem Territorium. Das Fürstentum umfaßte die Küstenstadt Sohar mit einem Teil der Batinah-Küstenebene und die im Landesinnern gelegene Stadt Rustaq mit den umliegenden Ländereien. Beherrscht wurde Sohar von einer Seitenlinie der in Oman regierenden Al-Bu-Said-Dynastie. Die Autonomie Sohars wurde vom omanischen Herrscher formell erst durch den Friedensvertrag von 1839 anerkannt, nachdem mehrere Versuche gescheitert waren, das Fürstentum gewaltsam in den Herrschaftsbereich von Oman wieder einzugliedern. Das Fürstentum bildet seit 1852 wieder einen festen Bestandteil Omans, nachdem der Herrscher von Sohar 1852 ohne direkte Erben gestorben war.

\section{1) 23. 12.1839}

Friedensvertrag zwischen Oman und Sohar, bestehend aus einer Präambel und 7 Artikeln. Die ersten 5 Artikel des auf britische Vermittlung am 23. 12. 1839 in Muscat unterzeichneten und sofort in Kraft getretenen Vertrages sind reziprok. Die beiden Parteien vereinbaren darin „a perfect, lasting and established peace“ und verpflichten sich, sich jeglicher offener oder heimlicher Angriffe auf die andere Partei zu enthalten und auch keine dritte Macht zu solchen Akten aufzuwiegeln. Es wird ferner vereinbart, daß die Untertanen der beiden vertragsschließenden Herrscher ohne Behinderung von einem Gebiet in das andere überwechseln und auch ihren Wohnsitz ohne Behinderung von einem Gebiet in das andere verlegen können. Verbrecher und Rebellen, die von einem Territorium ins andere fliehen, sollen dort weder offene noch heimliche Unterstützung finden. In Artikel 6 garantiert der omanische Herrscher, daß er den freien Zugang von Sohar in das von omanischem Gebiet eingeschlossene Rustaq gewährleisten werde. In Artikel 7 verpflichtet sich der omanische Herrscher, Sohar im Falle eines Angriffes durch eine dritte Macht zu unterstützen ,in every way to the utmost of his power and ability“. 
Der Vertrag wurde zwar verschiedentlich von beiden Seiten gebrochen, bildete aber im wesentlichen die Grundlage für das beiderseitige Verhältnis bis 1852. Die Eigenständigkeit von Sohar wird noch durch ein Übereinkommen zwischen Sohar und Großbritannien vom 22. 5. 1849 zur Bekämpfung des Sklavenhandels unterstrichen. (Text: Aitchison ${ }^{1}$, Bd. 7, S. 111). Hinweise auf das Fürstentum Sohar siehe MILES; PHILLIPS, S. 100-101; WILSON, S. 217; LANDEN, S. 70.

\section{SÜDAFRIKA (NATAL)}

Natal erhielt schon früh weitgehende Selbstverwaltung und hatte ab 1893 volle Selbstregierung. Seit 1910 ist Natal eine Provinz der Südafrikanischen Union. Zwischen Natal bzw. der Südafrikanischen Union und Oman wurden keine direkten Verträge abgeschlossen. Natal schloß sich dem Vertrag Omans mit Großbritannien vom 19. 3. 1891 am 23. 10. 1892 an (siehe HERTSLET'S, Vol. 19, S. 751). Als Natal als Provinz in die Südafrikanische Union eingegliedert wurde, übernahm die Südafrikanische Union das bestehende Vertragsverhältnis mit Oman. Das Vertragsverhältnis wurde von der Südafrikanischen Union mit Wirkung vom 27. 10. 1932 gekündigt. Im übrigen gilt das bei Irland gesagte. In der Verlängerung vom 11. 2. 1932 heißt es ausdrücklich: "It is also understood that it shall be open to the Dominion of Canada, the Union of South Africa (in respect of Natal, the Transvaal and the Orange Free State) and the Irish Free State to withdraw from the aforesaid treaty at any time on notice being given to that effect by His Majesty's representative at Muscat."

Die Südafrikanische Union beendete das Vertragsverhältnis mit Wirkung vom 27. 10. 1932, als der brit. Konsul in einem Brief No. C/188 auf Verlangen der Regierung der Südafrikanischen Union der omanischen Regierung davon Mitteilung machte. Der „withdrawal“ der Südafrikanischen Union wird im Vertrag von 1933 ausdrücklich dokumentiert: "It is also understood that the Commonwealth of Australia and the Union of South Africa (in respect of Natal, the Transvaal and the Orange Free State) have withdrawn from participartion in the aforesaid treaty as per notices given by His Majesty's representative at Muscat in his letters Nos. 6 and C/188 dated the 3rd December, 1923, and the 27th October 1932, respectively." Insgesamt bestanden also vom 23. 10. 1892 bis zum 27. 10. 1932 vertragliche Beziehungen zwischen Oman auf der einen und Natal bzw. der Südafrikanischen Union auf der anderen Seite.

\section{VEREINIGTE STAATEN VON AMERIKA (USA)}

\section{1) 21. 9. 1833}

Grundlegender Handels- und Freundschaftsvertrag zwischen Oman und den USA, bestehend aus 9 Artikeln. Der Vertrag wurde zum Prototyp für die folgenden Verträge Omans mit Großbritannien (1839) und Frankreich (1844). Einer der Artikel ist reziprok und gewährt den Staatsangehörigen der vertragsschließenden Länder Meistbegünstigungen in bezug auf Zölle und andere Gebühren. Die anderen Artikel sind unilateral und garantieren den USA verschiedene Handelsprivilegien einschließlich exterritorialer Rechte sowie das Recht zur Ernennung eines Konsuls. Der Vertrag wurde am 21. 9. 1833 von Saiyid Said, dem omanischen Herrscher, und Edmund Roberts, „being duly appointed a Special Agent by Letters Patent 
under the signature of the President, and seal of the USA bearing date at the City of Washington, the 26th January, for concluding a Treaty of Amity and Commerce between the USA and His Majesty Syud Sueed Bin Sultan, of Maskat", in Muscat in einer arabischen und einer englischen Version unterzeichnet. Die Ratifikation wurde am 23. 6. 1834 vom US-Senat gebilligt, am 30. 9. 1835 wurden die Ratifikationsurkunden in Muscat ausgetauscht, am 7. 4. 1834 wurde Robert H. Waters zum US-Konsul in Zanzibar ernannt und am 24. 6. 1834 wurde das Inkrafttreten des Vertrags durch Proklamation des US-Präsidenten bekanntgegeben.

Der Vertrag wurde durch einen neueren von 1958 ersetzt. Die USA unterhielten von 1837 bis 1856 ein Konsulat in Zanzibar und von 1880 bis 1915 (nach anderen Angaben 1916) ein Konsulat in Muscat. Sehr detaillierte Ausführungen über das Verhältnis Oman-USA und die wiederholten Versuche Omans, den Vertrag von 1833 teilweise zu revidieren, siehe GRAY, S. 194-223. Weitere Hinweise siehe PHILLIPS, S. 105-108; WILSON, S. 234 und 237; und UN DOC A/5846, $§ 407$.

\section{2) 20.12. 1958}

Grundlegender Freundschafts-, Wirtschafts- und Konsularrechtsvertrag zwischen Oman und den USA, bestehend aus einer Präambel und 16 Artikeln. Der Vertrag ersetzt mit seinem Inkrafttreten den Vertrag von 1833 (s. Art. 15) und enthält detaillierte Bestimmungen - alle reziprok - über die Beziehungen zwischen beiden Staaten. Der Vertrag wurde am 20. 12. 1958 in Salalah unterzeichnet, am 19. 2. 1959 mit der Bitte um Weiterleitung an den US-Senat und um Ratifikation dem USPräsidenten übermittelt, am 7. 4. 1959 vom zuständigen Komittee dem Senat zur Ratifikation empfohlen, am 8. 5. 1959 vom Präsidenten ratifiziert, und am 11. 5. 1959 wurden die Ratifikationsurkunden ausgetauscht. Danach trat der Vertrag am 11.6.1960 in Kraft. .

\section{MULTILATERALE VERTRÄGE}

1) Beitrittserklärung Omans zur "International Convention and Protocol relative to the Control of the Trade in Arms and Ammunition, signed at Saint-Germainen-Laye, September 10, 1919." Der Sultan von Oman übermittelte seine Beitrittserklärung am 17. 2. 1921 in einem Brief an den britischen Konsul in Muscat und beauftragte Großbritannien, die Erklärung bei der französischen Regierung, dem Depositär der diesbezüglichen Urkunden, hinterlegen zu lassen. Der Beitritt Omans wurde am 9. 6. 1921 anerkannt und bekanntgegeben. Der Beitritt zu dieser Konvention bildete den Abschluß der omanischen Bemühungen, auf britischen Wunsch und im eigenen Interesse den illegalen Waffenhandel, dessen Metropole Muscat war, zu unterdrücken. Hinweis siehe ferner PHILLIPS, S. 162.

2) Im Mai 1971 wurde Oman als 130. Mitglied (mit 95 Stimmen gegen 2, bei 10 Enthaltungen) in die Weltgesundheitsorganisation aufgenommen, so daß es nunmehr der Satzung der WHO vom 22. 7.1946 und der Internationalen Gesundheitsordnung vom 25. 5. 1951 untersteht. 


\section{Chronologische Übersicht und Fundstellenverzeichnis}

Die Daten in Klammern bedeuten das Inkrafttreten. Wo kein solches Datum vorkommt, ist der Vertrag wahrscheinlich sofort in Kraft getreten.

1790 Briefwechsel über Beziehungen zwischen Frankreich und Oman (erwähnt bei Miles, S. 277/78)

1798 Vertrag Omans mit Persien über die Erneuerung der Verpachtung von Bandar Abbas, Oishn und Hormuz an Oman (erwähnt bei Miles, Hurewitz, Bd. 1, S. 157, u. a.)

12. 10. 1798 Schriftliche Verpflichtungserklärung Omans gegenüber Großbritannien zum Ausschluß von Franzosen

Engl. Text: Aitchison ${ }^{1}$, Bd. 7, S. 87

Aitchison ${ }^{5}$, Bd. 11, S. 287

State Papers, Bd. 32, S. 188

Moyse-Bartlett, S. 235

Hurewitz, Bd. 1, S. 64

18. 1. 1800 Übereinkommen mit Großbritannien (Indien) betr. Installierung eines brit. Agenten in Muscat. Ratifiziert vom Vizekönig von Indien am 26. 4. 1800

Engl. Text: Aitchison 1 , Bd. 7, S. 88

Aitchison ${ }^{5}$, Bd. 11, S. 288

State Papers, Bd. 32, S. 189

Moyse-Bartlett, S. 237

1803 Waffenstillstandsvertrag für drei Jahre zwischen Oman und dem Emir der Wahabiten (erwähnt bei Miles)

16. 6. 1807 Friedensvertrag des Kaisers der Franzosen mit Oman (erwähnt bei Gray, S. 180, und Miles)

17.6. 1808 Konvention mit Frankreich zur Änderung des Vertrages von 1807 (erwähnt bei Gray, S. 180, und Miles)

April 1817 Firman des Schahs von Persien betr. Zusage zur Unterstützung bei der Eroberung von Bahrain (erwähnt bei Adamiyat)

März 1820 Vertrag mit dem Scheich von Bahrain (erwähnt bei Adamiyat)

4. 9./9./10. 9.1822 Vertrag mit Großbritannien über Abschaffung der Sklaverei

(10.9. 1822) Zusatz v. 9.9.1822

Verkündet durch Proklamation vom 30. 10. 1822

Engl. Text: Aitchison ${ }^{1}$, Bd. 7, S. 89 (Zusatz S. 91)

Aitchison ${ }^{5}$, Bd. 11, S.

Hertslet's, Bd. 3, S. 265 (Prokl. S. 269)

State Papers, Bd. 12, S. 464

Febr. 1826 Arrangement mit dem Generalgouverneur von Portug. Mozambique betr. Handel, Jurisdiktion, Wracks usw.

Engl. Text: State Papers, Bd. 73, S. 1176

28. 3. 1828 Handels-, Schiffahrts- und Freundschaftsvertrag mit Portugal (von Oman nicht ratifiziert!)

Port. Text: Mozambique Pasta, Bd. 4, 1840

27. 5. 1829 Grundsatzvertrag mit Portugal

(von Oman und Portugal nicht ratifiziert!)

(erwähnt bei Gray, S. 176)

2. 12. 1829 Friedensvertrag mit Bahrain

(erwähnt bei Miles) 
Mai 1833 Vertrag mit dem Emir der Wahabiten (Saudi-Arabien)

(erwähnt bei Kelly, S. 62, und bei Miles)

21. 9. 1833 Freundschafts- und Handelsvertrag mit den USA

(30. 6. 1834) Proklamation der USA: 24. 6. 1834

Amtl. Text: Statutes, Bd. 8, S. 458 (TS 247)

Engl. Text: Aitchison ${ }^{1}$, Bd. 7, App. S. CLXXXVI

Hurewitz, Bd. 1, S. 108 (danach Ratif. anst. 30. 9. 35)

State Papers, Bd. 22, S. 587

Malloy, S. 1228

1836 Allianz zwischen Oman und dem Befehlshaber der Wahabiten in Buraimi zur Rückeroberung von Sohar (erwähnt bei Miles)

um 1839 Protektionsvertrag mit Nossi Bé (erwähnt bei Gray, S. 181)

31. 5. 1839 Handelsvertrag mit Großbritannien mit beiderseitigen Erklärungen bei der Ratifizierung vom 22. 7. 1840. Für Zanzibar aufgehoben am 3. 4. 1911 (State Papers, Bd. 104, S. 205) Engl. Text: Aitchison' ${ }^{1}$ Bd. 7, S. 93 (Erkl. S. 97)

Aitchison ${ }^{5}$, Bd. 11, S. 292

Hertslet's, Bd. 5, S. 611 (Erkl. S. 617)

State Papers, Bd. 28, S. 1080

17. 12. 1839 Zusatz-Artikel zum Vertrag mit Großbritannien von 1822

(Anfügung von drei neuen Artikeln)

Engl. Text: Aitchison ${ }^{1}$, Bd. 7, S. 99

Aitchison ${ }^{5}$, Bd. 11, S.

Hertslet's, Bd. 6, S. 578

State Papers, Bd. 29, S. 1109

23. 12. 1839 Friedensvertrag mit Sohar

Engl. Text: Aitchison, Bd. 7, S. 110

29. 9./20. 11. 1843 Abkommen mit Portugal betr. Papiere omanischer Schiffe, die portugiesische Häfen anlaufen

Port. Text: Mozambique Pasta, Bd. 6 (1842/43)

17. 11. 1844 Freundschafts- und Handelsvertrag mit Frankreich

Bei Ratifizierung am 4. 2. 46 auch Unterzeichnung eines Zusatz-Memorandums. Zum Vertrag vergl. franz. Gesetz betr. Konsulargerichtsbarkeit in Oman vom 8. 7. 1852 (State $\mathrm{Pa}$ pers, Bd. 72, S. 243)

Amtl. Text: J. O. v. 1. 8. 1846 (Kgl. Ordonnanz v. 22. 7. 1846

betr. Veröff. des Vertrages)

B. L. Nr. 12889

Franz. Text:

State Papers, Bd. 35, S. 1011

Dalloz 1846 III, S. 133

De Clercq, Bd. 5, S. 259

Engl. Text: Hurewitz, Bd. 1, S. 127

Aitchison ${ }^{1}$, Bd. 7, App. SCLXXXIX

Aitchison ${ }^{5}$, Bd. 11, App. S. XXIX

Febr. 1845 Waffenstillstand mit dem Befehlshaber der Wahabiten in Buraimi (erwähnt bei Kelly, S. 68, und Miles)

1845 Vertrag über Tribut Omans an die Wahabiten (erwähnt bei Kelly, S. 69, und Miles)

18. 8. 1845 Brief Omans an Großbritannien betr. Art. 4 des Vertrages von 1822

Engl. Text: Aitchison ${ }^{1}$, Bd. 7, S. 92 
2. 10. 1845 Übereinkommen mit Großbritannien betr. Beendigung des

(1. 1. 1847) Exports v. Sklaven aus den afrikanischen Besitzungen Omans Amtl. Text: 11/2 Vict. c. 128 v. 15. 9. 1848 (Brit. Ausfuhrges.) Engl. Text: Aitchison ${ }^{1}$, Bd. 7, S. $101=$ App. S.CXLII (Gesetz v. 1848)

Hertslet's Bd. 7, S. $818=$ Bd. 8, S. 753 (Gesetz v. 1848) State Papers, Bd. 35, S. 632

6. 5. 1850 Úbereinkommen mit Großbritannien betr. Sklavenhandel Engl. Text: Hertslet's, Bd. 9, S. 557

April 1853 Vertrag mit dem Befehlshaber der Wahabiten in Buraimi (erwähnt bei Kelly, S. 75, und Miles)

14.7.1854 Abtretungserklärung betr. die Kuria-Muria-Inseln an Großbritannien

Engl. Text: Aitchison ${ }^{1}$, Bd. 7, S. 102

Aitchison ${ }^{5}$, Bd. 11, S. 302

Hertslet's, Bd. 10, S. 938

Hurewitz, Bd. 1, S. 145

April 1856 Abkommen mit Persien über die Verpachtung von BandarAbbas usw. an Oman

Engl. Text: Badger, Einleitung

17. 11. 1856 Abkommen mit Persien über die Verpachtung von BandarAbbas usw. an Oman*

Engl. Text: Hertslet's, Persia Treaties, S. 112

Hurewitz, Bd. 1, S. 157

2. 4./15. 5. 1861 Briefwechsel Omans mit Großbritannien (Indien) betr. Zanzibar

Engl. Text: Aitchison'1, Bd. 7, S. 103

State Papers, Bd. 56, S. 1396

Wilson, S. 234 (Fußnote: Auszüge)

Kumar (Auszüge)

17./18. 11. 1864 Vertrag mit Großbritannien betr. Telegrafenkonzessionen Engl. Text: Aitchison ${ }^{1}$, Bd. 71, S. 105

19. 1. 1865 Konvention mit Großbritannien betr. Ausdehnung der Telegrafen in Arabien und Mekran Engl. Text: Aitchison'1, Bd. 7, S. 107

4. 8. 1868 Übereinkommen mit Persien über die Verpachtung von Bandar-Abbas an Oman

Engl. Text: Aitchison ${ }^{1}$, Bd. 7, App. S.CXXXVII

Aitchison ${ }^{4}$, Bd. 12, S. 113

1872 Vertrag mit Abu Dhabi über gemeinsame Verteidigung und Verwaltung von Buraimi (erwähnt: Phillips, S. 166; Landen, S. 326; Kelly, S. 100)

14. 4. 1873 Vertrag mit Großbritannien zur Abschaffung des Sklaven(Sept. 1873) handels

Engl. Text: Aitchison'1, Bd. 7, S. 108

State Papers, Bd. 63, S. 172

1873 Übereinkommen mit Großbritannien über Jurisdiktion über Angehörige indischer Schutzstaaten in Oman

Engl. Text: Aitchison'1, Bd. 7, S. 109

- Uber die Zweifel am Bestehen dieses Vertrages siehe oben unter Iran Nr. 4a. Eine weitere Version ist abgedrudkt bei Aitchison ${ }^{1}$, Bd. 7, App. CXXXV, datiert als Order von 1855. 
2./10. 2. 1875 Notenwechsel mit Großbritannien betr. Zoll Engl. Text: Aitchison'1, Bd. 7, S. 110

7. 4./27. 8. 1877 Notenwechsel (Deklaration) mit den Niederlanden betr. Handel

(27. 8. 1877) Amtl. Text: Staatscourant 1877, S. 259 (niederl.)

Tractatenblad v. 22. 5. 1969, Nr. 72, S. 1 (franz. u. niederl.)

Franz. Text: Aitchison 5 , Bd. 11, App. S. XXXIX

Lagemans, Reineil, Bd. 7, S. 603

Martens ${ }^{2}$, Bd. 4, S. 352

State Papers, Bd. 68, S. 36

19. 3. 1891 Freundschafts-, Handels- und Schiffahrtsvertrag mit Großbritannien

Unterzeichnung eines Zusatzprotokolls beim Austausch der Ratifikationen in Muscat am 20. 2. 1892

(20.3. 1892) 15.12.1892 Beitritt von Queensland (gekündigt v. Australien am 3. 12. 1923: State Papers, Bd. 117, S. 335)

15. 12. 1892 Beitritt von Neufundland

23. 10.1892 Beitritt von Natal (gekündigt von Südafrika am 27. 10. 1932)

11. 4.1893 Beitritt von Kanada

Verlängerungen (teils mit Zusatzerklärungen, wie Erstrekkung), ab 1919 je für ein Jahr, am 27. 11. 1937 bis zum 11. 2. 1939:

11. 2. 1914 State Papers, Bd. 112, S. 755

11. 2. 1919 State Papers, Bd. 112, S. 756

11. 2.1920 State Papers,

Bd. 113, S. 371 = LNTS, Bd. 5, S. 59

11. 2. 1921 State Papers,

Bd. 114, S. $345=$ LNTS, Bd. 8, S. 261

11. 2. 1922 State Papers,

Bd. 116, S. 503 = LNTS, Bd. 10, S. 459

11. 2. 1923 State Papers,

Bd. 117, S. 336 = LNTS, Bd. 17, S. 163

11. 2.1924 State Papers,

Bd. 119, S. 447 = LNTS, Bd. 25, S. 387

11. 2. 1925 State Papers,

Bd. 121, S. 816 = LNTS, Bd. 35, S. 233

11. 2. 1926 State Papers,

Bd. 123, S. $544=$ LNTS, Bd. 57, S. 13

11. 2.1927 State Papers,

Bd. 126, S. $269=$ LNTS, Bd. 64, S. 397

11. 2. 1928 (State Papers, Bd. 128, 322 Note)

LNTS, Bd. 83, S. 440

11. 2. 1929 (State Papers, Bd. 130, 171 Note)

LNTS, Bd. 96, S. 194

11. 2. 1930 (State Papers, Bd. 132, 305 Note)

LNTS, Bd. 100, S. 224

11. 2. 1931 State Papers,

134, S. $284=$ LNTS, Bd. 107, S. 499 
11. 2.1932 State Papers,

Bd. 135 , S. 296 = LNTS, Bd. 130, S. 444

11. 2. 1933 State Papers,

Bd. 136, S. 411 = LNTS, Bd. 138, S. 440

11. 2.1934 State Papers,

Bd. 137, S. 176 = LNTS, Bd. 156, S. 201

11. 2. 1935 (State Papers, Bd. 139, S. 204 Note)

LNTS, Bd. 160, S. 343

11. 2.1936 (State Papers, Bd. 141, S. 399 Note)

LNTS, Bd. 168, S. 230

11. 2. 1937 (State Papers, Bd. 141, S. 399 Note)

LNTS, Bd. 181, S. 365

27. 11. 1937 (State Papers, Bd. 142, S. 156 Note)

LNTS, Bd. 189, S. 459

Engl. Text: Aitchison ${ }^{5}$, Bd. 11, S. 310

State Papers, Bd. 83, S. 11

Hertslet's, Bd. 19, S. 745

Martens', Bd. 18, S. 636

20. 3. 1891 Übereinkommen mit Großbritannien betr. Nichtabtretung

(23. 5. 1891) von Gebiet (endet 1958, s. u.). Ratifiziert v. Vicekönig von Indien am 23. 5. 1891

Engl. Text: State Papers, Bd. 100, S. 591

Aitchison ${ }^{5}$, Bd. 11, S. 317

Martens ${ }^{3}$, Bd. 6, S. 287

Hurewitz, Bd. 1, S. 208

1896 Übereinkommen mit Abu Dhabi betr. gemeinsame Verwaltung und Aufrechterhaltung von Frieden und Sicherheit in Buraimi und Dhahirah (erwähnt: Phillips, S. 166, Kelly, S. 101, Lorimer I, S. 747)

10. 9. 1919 Internationale Konvention und Protokoll zur Kontrolle des

(9. 6. 1921) Handels mit Waffen und Munition

Beitritt Omans durch Brief an den britischen Konsul vom 17. 12. 1921

(Text: Aitchison und UN Doc A/5846, \417) am 9. 6. 1921 anerkannt.

Amtl. Text: LNTS, Bd. 7, S. 332 (Beitritt: Bd. 24, S. 158)

Engl. Text: State Papers, Bd. 112, S. 909 (Beitritt: Bd. 114, S. 549)

1934 Abkommen über Zivilluftfahrt mit Großbritannien (s. UN/ Doc. A/5846, \$607, und Annex VII, $\$ 13$ )

5. 2. 1939 Handels- und Schiffahrtsvertrag mit Großbritannien, gekün-

(11. 2. 1939) digt von Oman auf den 11. 2. 1951, aber dann zweimal verlängert bis 31. 12.1951 (UNTS, Bd. 149, S. 430)

Amtl. Text: LNTS, Bd. 196, S. 303, Cmnd 6037 (TS 1939, Nr. 29)

Engl. Text: State Papers, Bd. 156, S. 660

5. 4. 1947 Abkommen über Zivilluftfahrt mit Großbritannien

(5. 4. 1947) Amtl. Text: UNTS, Bd. 27, S. 287

Engl. Text: State Papers, Bd. 147, S. 928 
20. 12. 1951 Freundschafts-, Handels- und Schiffahrtsvertrag mit Großbri-

(19. 5. 1952) tannien mit Notenwechsel als Anlage (Jurisdiktion)

Amtl. Text: UNTS, Bd. 149, S. 247

Cmnd 8633 (TS 1952, Nr. 44)

Engl. Text: State Papers, Bd. 158, S. 348 (Briefe: S. 358), UN.Doc. A/6300/Rev. 1 (Annex)

26. 10. 1952 Waffenstillstand zwischen Saudi-Arabien und Großbritannien, letzteres handelnd für Abu Dhabi und für Oman (erwähnt bei Kelly, S. 159, Phillips, S. 170)

15. 3. 1953 Freundschafts-, Handels- und Schiffahrtsvertrag mit Indien

(2.3. 1954) Amtl. Text: UNTS, Bd. 190, S. 69

Engl. 'T'ext: State Papers, Bd. 160, S. 571

30. 7. 1954 Schiedsabkommen zwischen Saudi-Arabien und Großbritannien, letzteres handelnd für Abu Dhabi und für Oman

Amtl. Text: Cmnd 9272 (TS 1954, Nr. 65)

UNTS, Bd. 201, S. 317

Engl. Text: Kelly, S. 281

25. 4. 57/10. 3. 58 Notenwechsel (Übereinkommen) mit Großbritannien zur Än-

(6. 2. 1958) derung des Notenwechsels zum Vertrag vom 20. 12. 1951

Übertragung der Jurisdiktion über Inder an Oman

Amtl. Text: UNTS, Bd. 305, S. 430

Engl. Text: State Papers, Bd. 163, S. 490

25.7. 1958 Notenwechsel mit Großbritannien betr. Streitkräfte des Sul-

(25. 7. 1958) tans, Zivilluftfahrt, RAF-Stützpunkte und wirtschaftl. Entwicklung

Amtl. Text: UNTS, Bd. 312, S. 347

Cmnd 507 (TS 1958, Nr. 28)

Engl. Text: State Papers, Bd. 163, S. 491

UN Doc. A/6300/Rev. 1 (Annex)

26. 7./18. 8. 58 Notenwechsel mit Großbritannien betr. Beendigung des Úber-

(26. 7. 1958) einkommens vom 20. 3. 1891 betr. Nichtabtretung (erwähnt in State Papers, Bd. 163, S. 490)

20.12. 1958 Vertrag über Freundschaft, Wirtschaftsbeziehungen und Kon-

(11. 6. 60) sularrecht mit den USA, mit Notenwechsel (Protokoll) über

Konsularbeziehungen

Amtl. Text: UNTS, Bd. 380, S. 181

UST, Bd. M, S. 1835

TIAS, Nr. 4530

Engl. Text: State Papers, Bd. 163, S. 941

8. 9. 1958 Vereinbarung mit Pakistan über die Abtretung von Gwadur an Pakistan (erwähnt: Phillips, S. 69, Landen, S. 363, State Papers, Bd. 163, S. 490; UN Doc. A/5846, \529)

19. 6. 1967 Notenwechsel mit Deutschland betr. Aufnahme konsularischer Beziehungen (nicht veröffentlicht)

15. 11. 1967 Vertrag mit Großbritannien über die Rückgabe der KuriaMuria-Inseln an Oman

Amtl. Text: Cmnd 3505 (TS 1968, Nr. 8)

31. 3. 1968 Übereinkommen mit Abu Dhabi betr. allg. Beziehungen (erwähnt in UN Doc. A/7623/Add 5 (Part. II), $\ 8$ und $\$ 15)$

9. 4./25. 8. 1968 Notenwechsel mit den Niederlanden betr. Konsularbeziehun(25. 8. 1968) gen

Amtl. Text: Tractatenblad v. 22. 5. 1969, Nr. 72, S. 3 (engl.)

27. 12. 1968 Notenwechsel mit Deutschland betr. Konsularbeziehungen 21. 5. 1969 (nicht veröffentlicht) 


\section{Quellen}

Adamiyat Fereydoun Bahrein Islands, New York 1955

Aitchison, C. U. "A Collection of Treaties, Engagements and Sanads Relating to India and Neighbouring Countries", 1. Aufl., Bd. VII, 1876, Calcutta; 4. Aufl. 1909, Bd. XII; 5. Aufl. 1933, Bd. XI

Al-Baharna $\quad$ Legal status of Arabian Gulf States, Manchester 1968

Badger, G. P. "History of the Imams and Seyyids of Oman, by Salil ibn (ed. and trans.) Razik, from AD 661-1856", Haklyut Society, London 1871; desgl. Burt Franklin, New York, 1871

Gray, J. "History of Zanzibar", Oxford University Press, London 1962

Hertslet, E. "Persia Treaties”, London, 1891

Hertslet's Hertslet's Commercial Treaties, London

Hurewitz, J. C. "Diplomacy in the Near and Middle East", Princeton, New Jersey, 1956, Bd. 1

Kelly, J. B.

"Eastern Arabian Frontiers", London 1964

Kumar, R.

„India and the Persian Gulf Region, 1858-1907. A study in British imperial policy", London 1965

Landen, R. G. “ “Oman Since 1956”, Princeton, New Jersey 1967

Lorimer, J. G., "Gazetteer of the Persian Gulf, Oman and Central Arabia", compiler

Calcutta, 1908-1915, Bd. 1 und 2

LNTS

League of Nation Treaty Series

Martens

Nouveau Recueil des Traités

Miles, S. B.

"The Countries and Tribes of the Persian Gulf", London, 1. Aufl. 1919; 2. Aufl. 1966

Mocambique Pasta Arquivo Histórico Ultramarino, Lisboa, Mocambique Pasta

Moyse-Bartlett, H. "The Pirates of Trucial Oman”, London 1966

Phillips, W. “Oman: A History”, London 1967

Said-Ruete, R. “Said bin Sultan, 1791-1856”, London 1929

S. I. Statutory Instruments, London

S.R.O. Statutory Rules and Orders, London

State Papers British and Foreign State Papers, London

VRU Verfassung und Recht in Übersee, Hamburg

Un Doc United Nations Document

UNTS United Nations Treaty Series

Wilson, A. T. “The Persian Gulf”, London, 1. Aufl. 1928; 2. Aufl. 1954 